\title{
The Significance of the Lingual Nerve During Periodontal/Implant Surgery
}

Hsun-Liang Chan, * Daylene J.M. Leong, * Jia-Hui Fu, * Chu-Yuan Yeh, * Nikolaos Tatarakis, ${ }^{*}$ and Hom-Lay Wang ${ }^{\dagger}$

Background: Understanding the position of the lingual nerve is important when performing third molar extractions and periodontal and implant surgeries in the mandible. The careless management of the lingual flap can potentially cause damage to the lingual nerve. The location of the lingual nerve in the third molar region was described in the literature; however, to our knowledge, its course mesial to the third molar region was not reported. The aim of this study is to identify and measure the location of lingual nerves in relation to mandibular teeth in fresh cadaver heads.

Methods: Thirty lingual nerves from 18 cadaver heads were dissected, and the vertical distance from the lingual nerve to the mid-lingual cemento-enamel junctions of mandibular molars and premolars and the position where the lingual nerve left the lingual plate and moved toward the tongue were determined. Two cadaver heads were randomly selected and exposed to cone-beam computed tomography (CBCT) scans after the insertion of a wrought wire into the nerve. The same vertical distance as the clinical measurement was determined and compared.

Results: Seventy-five percent of lingual nerves turned toward the tongue at the first and second molar region. The vertical distance was 9.6,13, and $14.8 \mathrm{~mm}$ at the second molar, first molar, and second premolar, respectively. The difference between clinical and CBCT measurements was $0.57 \pm 2.62 \mathrm{~mm}$.

Conclusions: The course of the lingual nerve in relation to posterior teeth was described. This information can help surgeons gain more understanding of the location of the lingual nerve and perform safe surgeries in the mandible. J Periodontol 2010;81:372-377.

\section{KEY WORDS}

Anatomy; cone-beam computed tomography; dental implants; lingual nerve.

\footnotetext{
* Graduate Periodontics, School of Dentistry, University of Michigan, Ann Arbor, MI.

$\dagger$ Graduate Periodontics, Department of Periodontics and Oral Medicine, School of Dentistry, University of Michigan.
}

$\mathrm{T}$ The lingual nerve is a branch of the mandibular nerve. This nerve provides sensory innervation to the mucous membranes of the anterior twothirds of the tongue and lingual tissues. It also carries the taste sensation of the anterior two-thirds of the tongue from the chorda tympani nerve. After it branches from the mandibular nerve, it passes between the medial surface of the mandibular ramus and the medial pterygoid muscle. Then, it runs beyond the anterior edge of the medial pterygoid muscle and descends toward the distal side of the third molar.

The proximity of this nerve to the mandibular third molar region is a concern when performing flap surgery in this area. A $0.6 \%$ to $2 \%$ incidence of lingual nerve injury was reported after third molar extraction. ${ }^{1-5}$ In the dental literature,,${ }^{6,7}$ its location in relation to the lingual plate in the third molar area was described. On average, it is located $3 \mathrm{~mm}$ apical to the osseous crest and $2 \mathrm{~mm}$ horizontally from the lingual cortical plate in the third molar area. ${ }^{6}$ However, in $17.6 \%$ of cases, the nerve may be situated at or above the crest of bone, lingual to the mandibular third molars. ${ }^{7}$ Furthermore, $22 \%$ of the time, the lingual nerve may contact the lingual cortical plate. ${ }^{6}$

To reduce the chance of injuring this nerve, some procedures have been advocated. ${ }^{8,9}$ For example, incisions distal to the third molar should be made on the buccal aspect of the ridge and always on the bone. The elevator should be used

doi: 10.1902/jop.2009.090506 
to protect the nerve in the flap, and the tissue should be managed gently. On the lingual side, it is also recommended that vertical releasing incisions should be avoided.

Although the position of the lingual nerve at the third molar region was studied, ${ }^{6,7,10,13}$ its course anterior to the third molar area, to the best of our knowledge, was not described. Understanding the position of the lingual nerve at the mandibular posterior region is important because of its superficial location and proximity to the lingual plate. Careless management of the lingual flap or the encroachment of the sublingual space may inevitably damage the lingual nerve, causing a temporary or permanent loss of lingual sensation. At least one case of lingual nerve paresthesia after implant placement ${ }^{14}$ and a case series $^{15}$ on trigeminal nerve injuries, including the lingual nerve, after dental treatment, were reported. Some articles $^{8,9,16,17}$ addressed safety issues with regards to implant or periodontal surgeries at the mandibular lingual region. Therefore, the aims of this study are to measure the vertical distances of the lingual nerve in relation to premolars and molars and to determine where the lingual nerve leaves the lingual plate and moves toward the tongue.

\section{MATERIALS AND METHODS}

\section{Clinical Measurements}

Eighteen fresh cadaver heads were studied. The cadaver heads were donated to the University of Michigan Anatomy Department for educational purposes; they were used for the present study after academic use. One examiner (HLC) dissected the lingual nerve and the other examiner (DJML) recorded and confirmed the position of the lingual nerve. The lingual nerve was dissected after a 2 -cm horizontal incision was made $3 \mathrm{~mm}$ apical to the osseous crest at the third molar site (Figs. 1 and 2). Every care was taken when dissecting the lingual nerve to not change the spatial relationship of the lingual nerve to the rest of the anatomies. The dissection was continued from the third molar area to the site where the lingual nerve started to leave toward the tongue. The position where the lingual nerve changed its course was recorded in relation to the tooth site. The vertical distances between the lingual nerve and the tooth, from second molars to the tooth where the lingual nerve curved toward the tongue, were measured using a University of North Carolina probe, ${ }^{\ddagger}$ which was accurate to $1 \mathrm{~mm}$. The measurements were made from the cementoenamel junctions (CEJs) at the mid-lingual sites to the superior edge of the lingual nerves. In cases of crowns, the measurements were made from the margins. The mean \pm SD was calculated for the measured variable.

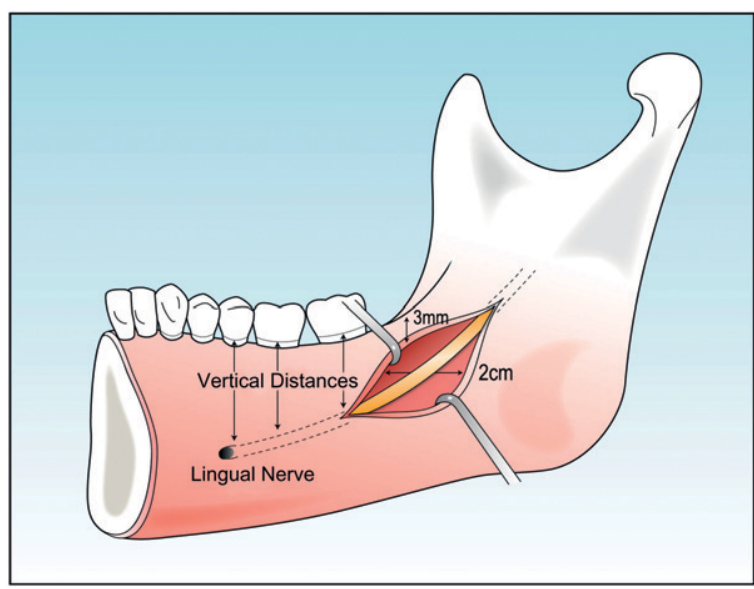

Figure I.

The technique of dissecting the lingual nerve and the measurement of the relationship between the nerve and mandibular posterior teeth.

\section{Cone-Beam Computed Tomography (CBCT) Measurements}

To confirm the clinical measurements, the position of the lingual nerve was determined on CBCT scans. Two specimens were randomly chosen for CBCT measurements. To visualize the lingual nerve in the images, a 0.012-in wrought wire was inserted into the lingual nerve sheath, as described by Karakas et al. ${ }^{10}$ and Trost et al. ${ }^{18}$ The wire was inserted from the third molar region until the curve was reached where the nerve entered the tongue. The nerve and tongue were passively placed back to their original position, and the specimens were exposed by a CBCT machine $\$$ in the Radiology Department of the University of Michigan. The specimens were stabilized using a head locator, and the scout view was obtained first to verify the region of interest. Subsequently, they were exposed to radiations with $120 \mathrm{KV}$ (peak) and 18.66 $\mathrm{mA}$ for 20 seconds. The resolution was set at $0.4 \mathrm{~mm}$, and the field of view was $16 \times 22 \mathrm{~cm}$. The data images were reconstructed using the built-in software packagell on a desktop computer. The position of the lingual nerve was measured on cross-sectional views chosen at the mid-lingual site of the respective tooth. The measurements were made from the CEJs or crown margins to the superior edge of the wire (Fig. 3).

\section{Statistical Analysis}

All data analyses were performed with statistical software. "I The agreement of the position where the lingual nerve left the lingual plate at both sides was presented with a cross table, and the $\kappa$ value was

† UNC-15 probe, Hu-Friedy, Chicago, IL.

$\S$ i-CAT Cone-Beam Computed Tomography machine, Imaging Sciences International, Hatfield, PA.

Invivodent, Anatomage, San Jose, CA.

II SPSS, SPSS version 12.0, Chicago, IL. 


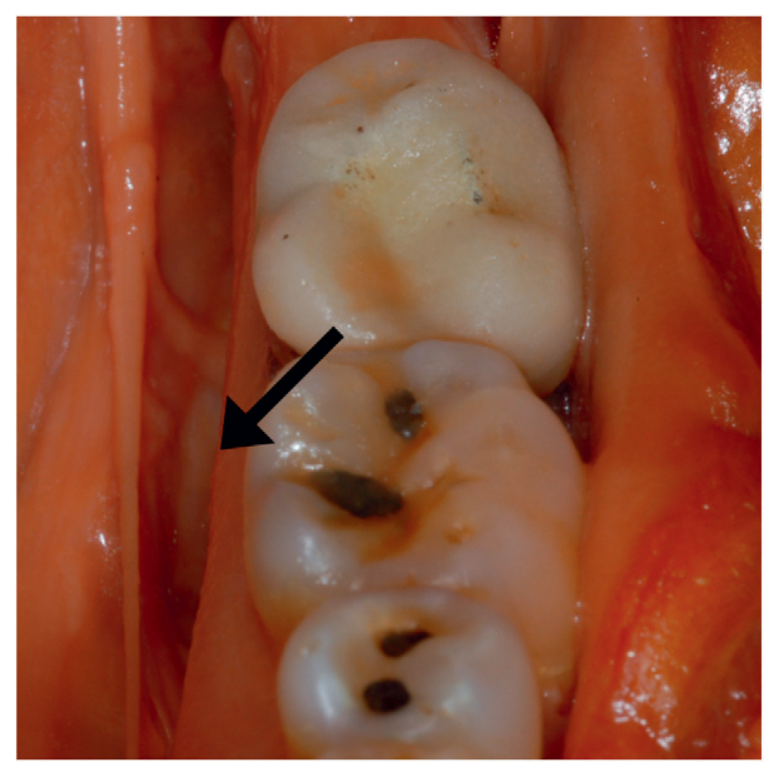

Figure 2.

Clinical photograph of the dissected lingual nerve (black arrow).

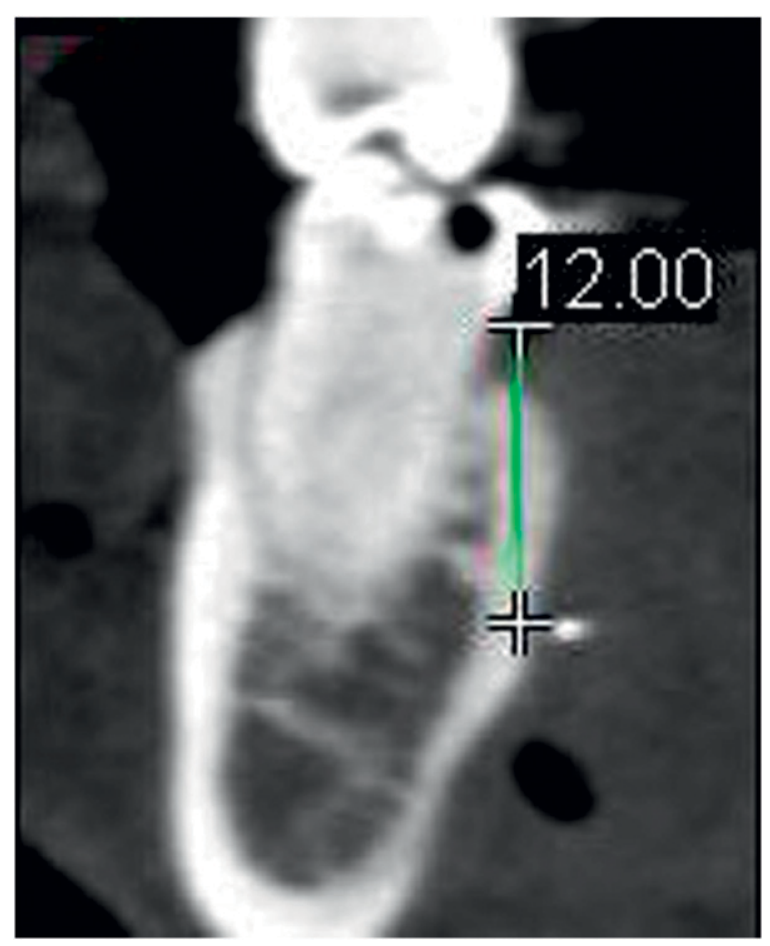

Figure 3.

$C B C T$ measurement of the location of the lingual nerve (the white dot indicates the cross-sectional view of the 0.012 -in wire inserted in the nerve). The vertical distance from the CEJ to the lingual nerve is $12 \mathrm{~mm}$ as shown. determined. Furthermore, the vertical position of the lingual nerve at both sides was compared using the Wilcoxon test. A $P$ value $<0.05$ was considered statistically significant. The differences between clinical and CBCT measurements were presented as the mean \pm SD (millimeters).

\section{RESULTS}

The study group was composed of 10 males and eight females (mean age: 70.2 years; age range: 33 to 97 years). A total of 30 nerves were studied because six unilateral edentulous mandibles precluded the measurements. Twelve pairs of lingual nerves were compared for the position where the lingual nerve left the lingual plate. On the right side, $41.67 \%$ (five of 12 nerves) of the lingual nerves curved toward the tongue at the first molar region. This occurred $33.33 \%$ and $25 \%$ at the second molar and second premolar regions, respectively. Furthermore, on the left side, $58.33 \%$ (seven of 12 nerves) of the lingual nerves left the lingual plate at the first molar region. Both second molar and second premolar sides accounted for $16.67 \%$ of the chance. One of 12 nerves (8.33\%) changed its course at the first premolar region. The $\kappa$ value was 0.51 , suggesting a high agreement between both sides (Table 1). The mean vertical distance of the lingual nerve at the right second molar, first molar, and second premolar was 9.5, 12.7, and $14.3 \mathrm{~mm}$ compared to $9.7,13.2$, and $15.7 \mathrm{~mm}$ at the contralateral side. There was no statistically significant difference in the vertical distance at second molar and first molar sites (both $P=0.89$ ). The vertical distances of the lingual nerve in the second and first premolar sites were not compared because of the limited sample size (Table 2). The nerve locations at six sites (four sites at the second molars and two at the first molars from two specimens) were compared for

\section{Table I.}

Agreement (12 pairs) in the Position Where the Lingual Nerve Left the Lingual Plate

\begin{tabular}{lccccc}
\hline Right/Left & $\begin{array}{c}\text { Second } \\
\text { Molar }\end{array}$ & $\begin{array}{c}\text { First } \\
\text { Molar }\end{array}$ & $\begin{array}{c}\text { Second } \\
\text { Premolar }\end{array}$ & $\begin{array}{c}\text { First } \\
\text { Premolar }\end{array}$ & Total \\
\hline Second molar & 2 & 1 & 1 & 0 & 4 \\
First molar & 0 & 5 & 0 & 0 & 5 \\
Second premolar & 0 & 1 & 1 & 1 & 3 \\
First premolar & 0 & 0 & 0 & 0 & 0 \\
Total & 2 & 7 & 2 & 1 & 12 \\
$\kappa=0.51$. & & & & & \\
\hline
\end{tabular}


Table 2.

\section{Vertical Position (mm) of 30 Lingual Nerves (15 at each side)}

\begin{tabular}{lcccccccc}
\hline \multirow{2}{*}{$\begin{array}{l}\text { Vertical } \\
\text { Position }\end{array}$} & \multicolumn{4}{c}{ Right Side } \\
\cline { 2 - 9 } & \multicolumn{3}{c}{ Second Molar } & First Molar Second Premolar & First Premolar & First Premolar & Second Premolar First Molar Second Molar \\
\hline Mean distance & 9.5 & 12.7 & 14.3 & N/A & 25.0 & 15.7 & 13.2 & 9.7 \\
$\mathrm{SD}$ & 3.9 & 3.7 & 3.6 & $\mathrm{~N} / \mathrm{A}$ & $\mathrm{N} / \mathrm{A}$ & 1.5 & 4.3 & 2.9 \\
Minimum & 3.0 & 7.0 & 8.0 & $\mathrm{~N} / \mathrm{A}$ & 25.0 & 14.0 & 7.0 & 3.0 \\
Maximum & 17.0 & 20.0 & 19.0 & $\mathrm{~N} / \mathrm{A}$ & 25.0 & 17.0 & 22.0 & 14.0 \\
$\mathrm{n}$ & 12 & 9 & 6 & 0 & 1 & 3 & 12 & 13 \\
\hline
\end{tabular}

The lingual nerve numbers do not add up to 30 (or 15 at each side) due to our inability to measure the lingual nerve position because of missing teeth.

the differences between the clinical and CBCT measurements. The mean difference was $0.57 \pm 2.62 \mathrm{~mm}$.

\section{DISCUSSION}

Mozsary and Middleton ${ }^{19}$ described the usual course of the lingual nerve as, "After leaving the third division of the trigeminal nerve, the lingual nerve descends in the pterygomandibular space almost on the surface of the mandible, anterior to the inferior alveolar and the mylohyoid nerves. It does not loop around the submandibular gland; instead it leaves the pterygomandibular space and runs superficially just under the mucosa and on the periosteum of the lingual plate of the alveolar process close to the gingival margin." The proximity of the lingual nerve to the mandibular posterior teeth has never been quantified. To our knowledge, this article is the first to describe the position of the lingual nerve in relation to the teeth mesial to the third molar region. The vertical position of the lingual nerve was located, on average, $9.6 \mathrm{~mm}$ apical to the mid-lingual CEJ of the second molar. As it moved anteriorly, the distance to the teeth became wider, from $\sim 13 \mathrm{~mm}$ at the first molar to $\sim 15 \mathrm{~mm}$ at the second premolar. The vertical position of the lingual nerve was considered symmetrical in this study group because there was no statistically significant difference in the vertical distance at the first and second molar regions between the two sides $(P=0.89)$. The measured distance was reasonable when deduced from the vertical distance of the lingual nerve at the third molar region reported in the literature. ${ }^{6,13,14}$ The mean distance from the alveolar crest at the third molar to the superior border of the lingual nerve ranges from $2.28^{7}$ to $8.32 \mathrm{~mm} .{ }^{13}$ One article with the largest sample size (430 fresh cadavers with 669 nerves) reported a mean distance of $3.01 \mathrm{~mm}$.

The site where the lingual nerve turned away from the lingual plate and ran toward the tongue occurred at $75 \%$ of first and second molar sites. This result is largely in agreement with Pogrel et al. ${ }^{13}$ who reported a mean distance of $27.7 \mathrm{~mm}$ from the retromolar pad to the location where the lingual nerve diverged from the lingual plate of the mandible. When the mesiodistal crown width of mandibular molars is estimated as $10 \mathrm{~mm},{ }^{20}$ the mean distance of $27.7 \mathrm{~mm}$ found in the study of Pogrel et al. ${ }^{13}$ falls into the first molar area, as shown in our study. The $\kappa$ value for the agreement of the position where the lingual nerve left the lingual plate in both sides was 0.51 , suggesting the symmetrical course of the lingual nerve at the left and right sides.

Several methods were attempted to measure the position of the lingual nerve. In most articles, ${ }^{6,7,13}$ the location of the lingual nerve was directly measured from dissected cadaver heads. Some authors used imaging tools, such as magnetic resonance imaging $(M R I)^{12}$ or ultrasound, ${ }^{21}$ and other authors ${ }^{10,18}$ used wires inserted into the lingual nerve sheath, exposed the specimens with radiation, and made measurements from radiographic films. There are advantages and disadvantages associated with each method. For example, a dissection is easy to perform but it may change the normal spatial position of the nerve if not properly prepared. Indirect methods, such as an MRI and ultrasound, may be useful because they can measure the in situ position of the nerve without dissection. However, the resolution of the imaging methods may prevent examiners from accurately identifying and measuring the nerve. Finally, the insertion of the wire and exposure of the specimen with radiation can provide a clear location of the nerve but have the same drawbacks as the dissection method. In this report, two methods, direct measurement after dissection of the specimen and the measurement from CBCT images after wire insertion, were performed and compared. Every care was made not to change the spatial relationship between the lingual nerve and the rest of the anatomies. In addition, when taking CBCT scans, the tongues of the cadaver heads were placed in a passive position to not change the 
locations of the nerves. The mean difference between the clinical and CBCT measurements was $0.57 \mathrm{~mm}$ with an SD of $2.62 \mathrm{~mm}$. The variations might have resulted from the rigidity of the wire, the positions of the heads, the movements of lingual nerves during clinical measurements, and the relatively small sample size.

Neurosensory alterations can occur after third molar extractions and periodontal and implant surgeries. The alterations may result from the compression, stretching, severing, or needle penetration of the nerves. Three levels of nerve injuries, in increasing severity, were classified. ${ }^{9}$ Neurapraxia describes a mild injury due to compression or prolonged traction of the nerve. Because the axons are intact, complete recovery of sensation can be expected in 4 weeks. Axonotmesis, which may be due to severe compression or traction of a nerve, results in damage of some of the axons and may take 5 to 11 weeks before signs of sensation return. The most severe one, neurotmesis, involves the total disruption of the nerve, and the prognosis is unfavorable. The consequences of nerve injuries may fall into one of the following categories: paresthesia (numb feeling, burning, and prickling), hypoesthesia (reduced feeling), hyperesthesia (increasing sensitivity), dysesthesia (painful sensation), or anesthesia (complete loss of feeling). ${ }^{9}$

The incidence of lingual nerve disturbances subsequent to third molar extractions was reported to be $0.5 \%$ to $2.1 \% .{ }^{1-5}$ In most cases, the sensory changes can reverse within 3 months. Lingual nerve damage after implant surgery was reported in a case report. ${ }^{14}$ In that case, full sensation returned 2 months after the removal of the implants, which were placed too lingually. In a case series ${ }^{15}$ with 163 consecutive patients who were referred with trigeminal nerve (inferior alveolar or lingual nerve) involvement after dental treatment, seven cases were associated with periodontal surgeries. Fourteen of the total 163 patients underwent surgical repair, two patients obtained good improvement in sensation, seven patients obtained some improvement, and five patients obtained no improvement.

Although lingual nerve damage is uncommon, we cannot neglect the importance of its anatomic location during periodontal and implant surgeries. It is especially true now as the number of implants being placed is increasing. From the results, a close relationship between the lingual bone plate and the lingual nerve at the posterior teeth region was identified, especially at the second molar region. The variation in the location of the lingual nerve among subjects was also recognized. Therefore, when performing surgeries at the posterior lingual region, intrasulcular incisions without vertical releasing incisions are recommended, especially at the molar region. When reflecting lingual flaps, full-thickness flaps should always be used and every care should be taken so as not to damage the flap as it may contain the nerve. In addition, the lingual flap should be gently retracted to avoid injuring the lingual nerve with a sharp instrument.

\section{CONCLUSIONS}

In this article, the course of the lingual nerve mesial to the third molar was described, which can help surgeons gain an understanding of the location of the lingual nerve and avoid unnecessary damage of the lingual nerve during procedures. The results of this study also highlight the importance of knowing the anatomy during periodontal and implant surgeries.

\section{ACKNOWLEDGMENTS}

The authors thank Dean Muller and his staff in the Anatomy Department, University of Michigan, for the organization and transportation of cadaver heads, Ms. Chia-Nin Wang at the Department of Biostatistics, School of Public Health, University of Michigan, for her advice on the statistical analyses, and Dr. Chu-Chun Hsiao, private practice, Taoyuan, Taiwan, for helping with data collection. This article was partially supported by the University of Michigan Periodontal Graduate Student Research Fund. The authors report no conflicts of interest related to this study.

\section{REFERENCES}

1. Hillerup S, Stoltze K. Lingual nerve injury in third molar surgery I. Observations on recovery of sensation with spontaneous healing. Int J Oral Maxillofac Surg 2007; 36:884-889.

2. Gomes AC, Vasconcelos BC, de Oliveira e Silva ED, da Silva LC. Lingual nerve damage after mandibular third molar surgery: A randomized clinical trial. J Oral Maxillofac Surg 2005;63:1443-1446.

3. Gülicher D, Gerlach KL. Sensory impairment of the lingual and inferior alveolar nerves following removal of impacted mandibular third molars. Int J Oral Maxillofac Surg 2001;30:306-312.

4. Bataineh AB. Sensory nerve impairment following mandibular third molar surgery. J Oral Maxillofac Surg 2001;59:1012-1017, discussion 17.

5. Valmaseda-Castellón E, Berini-Aytés L, Gay-Escoda C. Lingual nerve damage after third lower molar surgical extraction. Oral Surg Oral Med Oral Pathol Oral Radiol Endod 2000;90:567-573.

6. Behnia H, Kheradvar A, Shahrokhi M. An anatomic study of the lingual nerve in the third molar region. J Oral Maxillofac Surg 2000;58:649-651; discussion 652-653.

7. Kiesselbach JE, Chamberlain JG. Clinical and anatomic observations on the relationship of the lingual nerve to the mandibular third molar region. $J$ Oral Maxillofac Surg 1984;42:565-567.

8. Greenstein G, Cavallaro J, Tarnow D. Practical application of anatomy for the dental implant surgeon. $J$ Periodontol 2008;79:1833-1846. 
9. Greenstein G, Cavallaro J, Romanos G, Tarnow D. Clinical recommendations for avoiding and managing surgical complications associated with implant dentistry: A review. J Periodontol 2008;79:1317-1329.

10. Karakas P, Uzel M, Koebke J. The relationship of the lingual nerve to the third molar region using radiographic imaging. Br Dent J 2007;203:29-31.

11. Hölzle FW, Wolff KD. Anatomic position of the lingual nerve in the mandibular third molar region with special consideration of an atrophied mandibular crest: An anatomical study. Int J Oral Maxillofac Surg 2001;30: 333-338.

12. Miloro M, Halkias LE, Slone HW, Chakeres DW. Assessment of the lingual nerve in the third molar region using magnetic resonance imaging. $J$ Oral Maxillofac Surg 1997;55:134-137.

13. Pogrel MA, Renaut A, Schmidt B, Ammar A. The relationship of the lingual nerve to the mandibular third molar region: An anatomic study. J Oral Maxillofac Surg 1995;53:1178-1181.

14. Berberi A, Le Breton G, Mani J, Woimant H, Nasseh I. Lingual paresthesia following surgical placement of implants: Report of a case. Int J Oral Maxillofac Implants 1993;8:580-582.

15. Pogrel MA, Thamby S. The etiology of altered sensation in the inferior alveolar, lingual, and mental nerves as a result of dental treatment. $J$ Calif Dent Assoc 1999;27:531, 534-538.

16. Hunt PR. Safety aspects of mandibular lingual surgery. J Periodontol 1976;47:224-229.

17. Reinhart TC. Re: Anatomic variation of the position of the lingual nerve. J Periodontol 1990;61:305-306.

18. Trost O, Kazemi A, Cheynel N, et al. Spatial relationships between lingual nerve and mandibular ramus: Original study method, clinical and educational applications. Surg Radiol Anat 2009;31:447-452.

19. Mozsary PG, Middleton RA. Microsurgical reconstruction of the lingual nerve. J Oral Maxillofac Surg 1984; 42:415-420.

20. Woelfel JB Sr. Dental Anatomy: Its Relevance to Dentistry, 5 ed. Hagerstown, MD: The Williams E Wilkins Company; 1997.

21. Olsen J, Papadaki M, Troulis M, Kaban L, O’Neill M, Donoff B. Using ultrasound to visualize the lingual nerve. J Oral Maxillofac Surg 2007;65:2295-2300.

Correspondence: Dr. Hom-Lay Wang, 1011 N. University Ave., Ann Arbor, MI 48109-1078. Fax: 734/936-0374; e-mail: homlay@umich.edu.

Submitted September 1, 2009; accepted for publication November 9, 2009. 